\title{
LA IMPORTANCIA DE LA POSICIÓN DEL CANDIDATO POLÍTICO EN EL TARJETÓN ELECTORAL SOBRE LA ATENCIÓN VISUAL
}

\author{
The importance of the political candidate's position on the ballot paper on the visual \\ attention
}

\author{
Marco David Alejandro Correa-Barrera \\ marcocoba@unisabana.edu.co \\ Laura Esther Mantilla-Mejía \\ lauramanme@unisabana.edu.co \\ Carlos José Salgado \\ carlos.salgado2@unisabana.edu.co \\ Universidad de La Sabana \\ Chía, Cundinamarca, Colombia
}

Administración de Mercadeo y Logística Internacionales (AMLI) NeuroSmartLab - Laboratorio de Neurociencias aplicadas al Marketing Facultad de la Escuela Internacional de Ciencias Económicas y Administrativas (EICEA)

\begin{abstract}
Resumen
Se examinó la posibilidad de identificar la relación entre la posición de los candidatos en los tarjetones electorales y la atención visual de los votantes. Para esto, se realizó una consulta de las relaciones ya establecidas entre el diseño de los tarjetones y la decisión de los votantes para posteriormente relacionar la influencia de la posición con el conocimiento previo acerca de los candidatos, y de esa manera poder establecer el patrón de posición dentro del tarjetón que incide en la mayor generación de atención visual dentro de los participantes. Para poder realizar esto se diseñó el tarjetón electoral considerando los siguientes factores: Candidatos con rostros generados aleatoriamente utilizando herramientas de inteligencia artificial, posición aleatorizada, tipografías, tamaños y colores estandarizados. Así pues, el tarjetón electoral (estímulo visual) se presentó aleatoriamente a participantes de entre 18 - 24 años en la sala de pruebas del NeuroSmartLab en el dispositivo que se tiene conectado al eye-tracker SMI Red $500 \mathrm{~Hz}$. A partir de los recorridos visuales grabados por el eye-tracker utilizando Ios programas SMI Experiment Center y SMI BeGaze se obtuvieron los Heat Maps, Focus Maps y los KPIs (indicadores clave de rendimiento por sus siglas en inglés) de las AOls (áreas de interés por sus siglas en inglés) donde se resaltan las fijaciones promedio, las secuencias y el tiempo de permanencia. Estos resultados proveen un acercamiento a que puede haber una influencia identificada en la atención visual frente a la posición del candidato en el tarjetón, siendo esto soportado por las fijaciones promedio y el tiempo de permanencia, ligándose a la posición central-izquierda del tarjetón. De manera preliminar se puede dar dicha conclusión que se enfoca únicamente en lo visual, sin embargo, para una futura orientación (complemento) se requiere de más resultados para un análisis de diferencia de medias que robustezca esta conclusión.
\end{abstract}

Palabras clave:

Eye-tracker, tarjetón electoral, candidato político, atención visual, posición. 
Introducción

El ejercicio de participación democrática es influenciado por diferentes factores que afectan, tanto la decisión de los votantes como los resultados respecto de los candidatos políticos. Así pues, factores visuales en el tarjetón electoral como: la tipografía, el tamaño de las casillas, los colores de los partidos políticos, y principalmente, la posición en la que se ubican los candidatos genera efectos perjudiciales para algunos y favorables para otros (Bagley, 1965; Blom-Hansen, Elklit, Serritzlew, \& Villadsen, 2016). Por tal motivo, se busca determinar si es posible precisar la existencia de una influencia derivada de la posición observada en el tarjetón en cuanto a las decisiones de los votantes ya que la literatura señala que la posición puede llegar a ser un factor determinante en multiplicidad de casos.

Debido al reducido número de investigaciones latinoamericanas referentes a los diferentes factores que pueden afectar o influenciar la decisión final de los votantes en las diferentes elecciones políticas, al realizar una investigación que especifique las diferentes variables que pueden jugar a favor o en contra de un candidato en cuanto a la posición mediante resultados provistos a manera de KPIs (Key Performance Indicator por sus siglas en inglés e Indicador Clave de Rendimiento en español), se aporta a la sociedad información valiosa y pertinente. Esto conlleva a enriquecer la disponibilidad de información referente a esta temática ya que estudios previos dentro de la revisión de literatura se han realizado en zonas con una cultura considerablemente distinta a la latinoamericana, y a partir de la investigación se puede establecer un acercamiento inicial a la importancia de la posición dentro del tarjetón y su incidencia en la posterior toma de decisiones.

\section{Método}

\section{Muestra}

Esta es una investigación considerada entre participantes dada la base potencial y actual de votantes, compuesta por jóvenes de entre 18 - 24 años de edad seleccionados de manera aleatoria para la realización del experimento en el eye-tracker SMI Red $500 \mathrm{~Hz}$ ubicado en el NeuroSmartLab de la Universidad de La Sabana (Chía, Cundinamarca, Colombia). Los participantes firmaron un consentimiento informado estándar previo al experimento dentro del aula de eye-tracker donde se encuentra únicamente el participante y el investigador, contándose de igual manera con la aprobación y supervisión del director del NeuroSmartLab. El experimento toma aproximadamente 60 - 90 segundos por participante, siendo de estos 4 segundos para la visualización del cada estímulo visual ( 6 tarjetones electorales) y los demás en lo que se hace la acomodación del participante en el espacio junto a la explicación del investigador del procedimiento a seguir.

\section{Aparatos y materiales}

El instrumento usado es el eye-tracker SMI Red $500 \mathrm{~Hz}$ del NeuroSmartLab donde se presenta a cada participante el estímulo visual que es el tarjetón electoral diseñado considerando los siguientes factores: Candidatos de ambos sexos cuyos rostros fueron generados de manera aleatoria utilizando una herramienta de inteligencia artificial, asimismo posicionados de manera aleatorizada dentro del tarjetón, usando tipografías y tamaños estandarizados, además de los colores blanco (RGB: \#FFFFFF) y negro (RGB: \#000000). Lo anterior permite que se reduzca la variabilidad de los factores que pueden incidir en la toma de decisión y el movimiento ocular del votante, y por ende en su atención visual. Se presentaron un total de 6 variaciones de tarjetones electorales. La figura 1 presenta el modelo del tarjetón electoral diseñado de manera estandarizada.

Figura 1. Tarjetón electoral. 


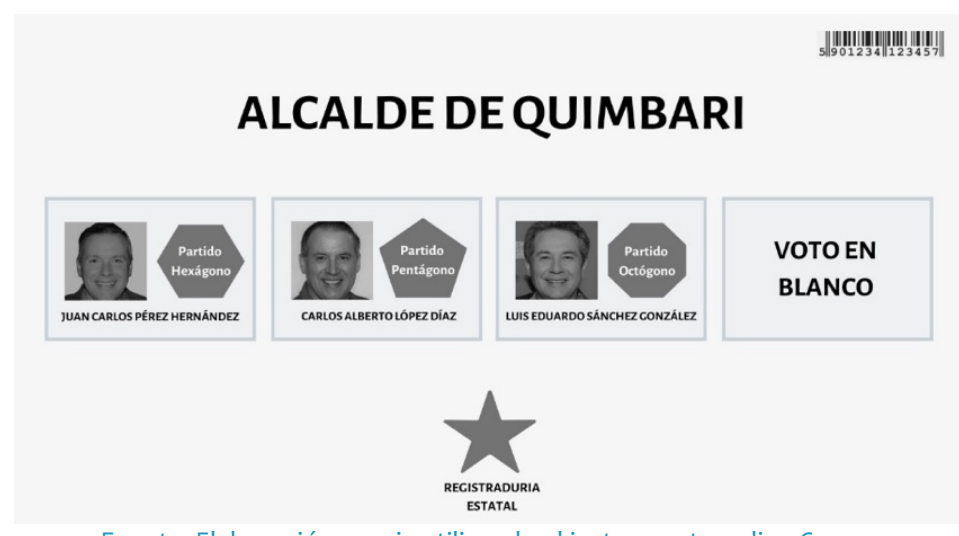

Fuente: Elaboración propia utilizando el instrumento online Canva.

\section{Procedimiento}

El procedimiento consistió en llevar de manera individual y aleatoria a cada uno de los participantes hacia la sala de pruebas del laboratorio en el que el estímulo visual fue mostrado en la pantalla del computador donde se encuentra ligado el eye-tracker SMI Red $500 \mathrm{~Hz}$ para así grabar el recorrido visual generado sobre el estímulo. El investigador brinda un consentimiento informado al participante para firmarlo junto a una breve explicación del tiempo para completar el experimento, así como los requerimientos para que éste se lleve a cabo sin mayor dilación. Estos tarjetones diseñados bajo todos los factores previamente detallados únicamente varían la posición del candidato y su aparición en pantalla es aleatorizada. La figura 2 resume el proceso metodológico realizado.

\section{Análisis de datos}

El software SMI Experiment Center fue utilizado para la obtención de los datos obtenidos por parte del eye-tracker SMI Red $500 \mathrm{~Hz}$, mientras que el software SMI BeGaze fue utilizado para el análisis de los resultados de una manera visual en términos de los KPIs de las AOIs (áreas de interés por sus siglas en inglés), de la concentración de miradas y de la totalidad de lo visualizado en los estímulos presentados (utilizando Heat Maps y Focus Maps). Entre los diferentes indicadores obtenidos se termina por dar una presentación de los más relevantes para cada una de las posiciones a evaluar de las que se presentan en el tarjetón electoral, siendo estos: fijaciones promedio, secuencia y tiempo de permanencia.

Figura 2. Proceso metodológico llevado a cabo.

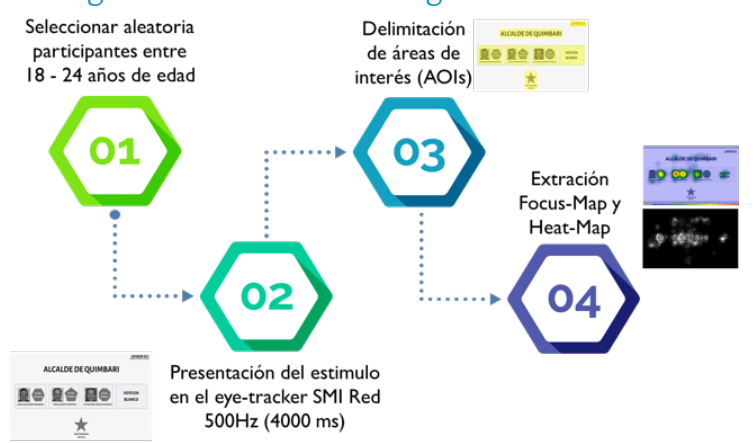

Fuente: Elaboración propia aunando el diseño preliminar del tarjetón y resultados visuales obtenidos del Eye-Tracker.

\section{Revisión de literatura}

A lo largo del tiempo, el posicionamiento de los candidatos dentro de los tarjetones de votación ha sido tema de discusión, puesto que, los votantes tienden a elegir a los que están en las primeras posiciones ya que cuanto más baja sea la posición de un candidato en el tarjetón tiene menos posibilidades de recibir atención o votos. (Robson \& Walsh, 1974; Alvarez, Sinclair \& Hasen, 2006; Jun 
\& Min, 2017; Grant, 2017). Esto se ve soportado por estudios realizados en lugares como Polonia y Bavaria (Alemania), donde la primera evidencia que dentro de los procesos electorales un candidato puede ser percibido como bien cualificado debido a su punto de relevancia en una lista (Marcinkiewicz, 2014), mientras que el segundo resalta que las posiciones más votadas tienden a ser las del cuadrante superior-izquierdo (Faas \& Schoen, 2006). Además, las influencias del primer puesto muestran que el efecto de la posición en la papeleta/tarjetón es causal ya que el orden de los nombres funciona como una señal para los votantes (Blom-Hansen, Elklit, Serritzlew, \& Villadsen, 2016) y entre más información tienen los votantes sobre los candidatos, menos influencia tiene la ubicación de estos en el tarjetón (Brockington, 2003).

Este efecto puede ser aún más notorio en una población indecisa con bajos niveles de información y en contextos en los cuales es obligatorio ejercer el derecho al voto, puesto que podría darse propensión a votar por los candidatos que ocupan el primer lugar de lista a la que adscriben (Quiroga \& Becerra, 2018). No obstante, el verdadero factor de incidencia viene a ser la atención visual ya que a pesar de existir estudios previos sobre la selección relacionada con la posición, no hay no hay estudios que expliquen o demuestren cómo se puede dar una afectación a la atención visual, la cual es una medida de importancia ya que por esta "se refiere a un conjunto de operaciones cognitivas que median la selección de información relevante y el filtrado de información irrelevante de escenas visuales desordenadas". (McMains \& Kastner, 2009). Asimismo, de acuerdo con Connor, Egeth \& Yantis cuenta con una atracción ligada a lo que es un estímulo saliente que sobresale de los alrededores y que, en ocasiones, puede tener una voluntariedad en cuanto a su orientación hacia los objetos dependiendo la importancia que este tenga para el observador, por ejemplo, si el participante conoce quién se encuentra en el tarjetón, se dará mayor atención visual en dicha sección del tarjetón (2004) (Camejo Ruiz, 2019; Daza-Orozco, 2019; Dos Santos Amaro Elías; Nogales Bocio, Antonia Isabel; Pérez Calle, Begoña; Pérez Lagos, Camila; Marta-Lazo, Carmen; Barredo Ibáñez, Daniel; De La Garza Montemayor, Daniel Javier; Murcia Quiñones, Harvey; Gil Quintana, J, Jenny Juliana, 2019; E. Norman-Acevedo, 2018; E Norman-Acevedo, 2019; Serrano Marín Rodrigo; Palencia Triana, César Augusto; Merino Jara, Claudio Esteban; Silva Echeto, Víctor; Villasana López, Pedro Enrique; Jiménez Díaz, Luis, 2019; Zabala-Leal, 2019).

\section{Resultados preliminares}

Con el desarrollo de la revisión de literatura se infiere que los candidatos que se encuentran ubicados en la primera posición presentan una ventaja frente a quienes están posicionados en puntos diferentes a este. A partir de los KPIs obtenidos de lo que se considera como AOIs con el programa BeGaze se da un análisis comparativo entre estos para así identificar el nivel de atención visual generado en cada uno, dado que el número de procesos complejos en el transcurso de la visualización del estímulo conlleva a que haya un reconocimiento del objeto de interés y se determine la guía del movimiento visual en este, asumiendo que al observar una escena visual meramente se está consciente de los elementos atractores y distractores de atención visual. Por ende, se ha de considerar que el participante no ha de conocer de manera previa el rostro de la persona que se encuentra dentro del tarjetón porque se evidencia que en caso de existencia de este conocimiento hay un condicionamiento e influencia a la hora de generar atención visual independientemente de su posición dentro del tarjetón y esto ya genera un proceso consciente del movimiento visual dentro del estímulo presentado. 
Figura 3. Heat Map del tarjetón electoral.

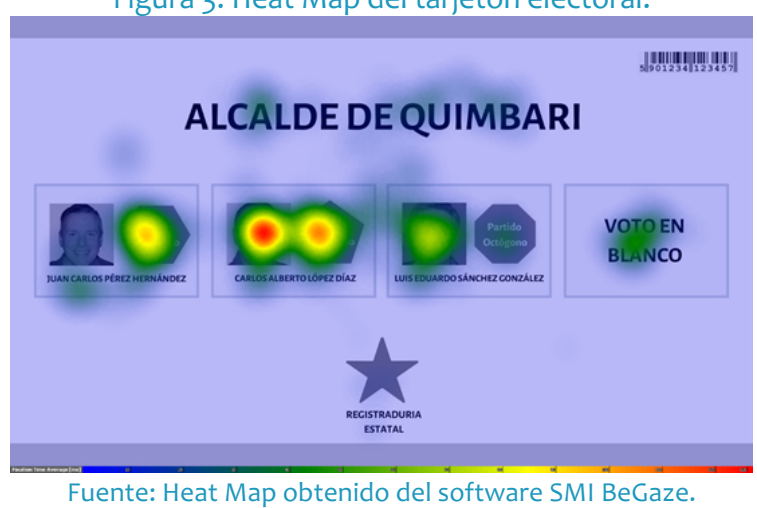

Figura 4. Focus Map del tarjetón electoral.

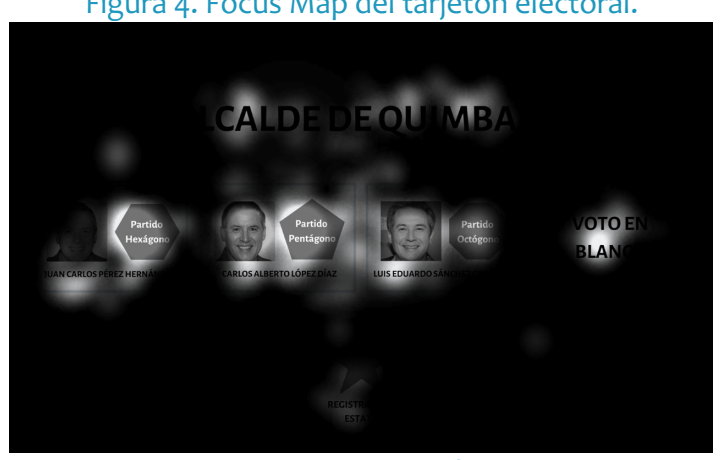

Fuente: Focus Map obtenido del software SMI BeGaze.

Dentro del Heat Map que presenta la concentración de la atención visual donde los colores más cálidos representan mayor concentración de miradas se puede denotar que hay efectivamente una concentración significativa en la parte superior izquierda; sin embargo, la parte más central que es la parte central-izquierda termina por obtener aún una mayor concentración que la previamente detallada, significando esto que sus fijaciones promedio y su tiempo de permanencia es mayor(AriasVelandia, Rincón-Báez, \& Cruz-Pulido, 2018; Benítez, Quintero, Márquez, \& Ayala, 2015; Oviedo \& Silva, 2017; Rigo \& Donolo, 2016). Asimismo, el Focus Map que presenta las áreas que efectivamente fueron observadas dentro del tarjetón electoral mientras que las áreas oscuras no obtuvieron recorridos visuales exhibe que dentro de las áreas marcadas la posición superior-izquierda obtiene mayor concentración de miradas en el partido político, mientras que la posición central-izquierda tanto en el partido como en el rostro del candidato político.

Tabla 1. Indicadores considerados dentro de las AOIs.

\begin{tabular}{|c|c|c|c|c|}
\hline & $\begin{array}{c}\text { Posición } \\
\text { superior-izquierda }\end{array}$ & $\begin{array}{c}\text { Posición } \\
\text { central-izquierda }\end{array}$ & $\begin{array}{c}\text { Posición } \\
\text { central-derecha }\end{array}$ & $\begin{array}{c}\text { Posición } \\
\text { superior-derecha }\end{array}$ \\
\cline { 2 - 5 } & Candidato I & Candidato 2 & Candidato 3 & Voto en blanco \\
\hline Fijaciones promedio & $125.9 \mathrm{~ms}$ & $186.9 \mathrm{~ms}$ & $120.1 \mathrm{~ms}$ & $81.7 \mathrm{~ms}$ \\
\hline Secuencia & 4 & $\mathrm{I}$ & 2 & 5 \\
\hline Tiempo de permanencia & $461.2 \mathrm{~ms}(\mathrm{I7.1} \%)$ & $726.4 \mathrm{~ms}(28.1 \%)$ & $376.0 \mathrm{~ms}(\mathrm{I} 4.6 \%)$ & I I6.9 ms (3.4\%) \\
\hline
\end{tabular}

Fuente: Elaboración propia aunando los resultados cuantitativos obtenidos del Eye-Tracker.

Finalmente, para presentarse la comparación de esto se cuenta con la tabla 1 donde se presentan por posición los indicadores de fijaciones promedio, la secuencia de visualización sobre cada una de estas áreas dentro del estímulo visual y el tiempo de permanencia que se obtuvo por parte de los participantes (votantes). Dentro de esta tabla se puede apreciar que -en concordancia con lo previamente presentado en el Heat Map y Focus Map- la posición central-izquierda es la que se 
observa en primera instancia, la que obtiene la mayor concentración de miradas y cuyas fijaciones promedio superan la posición superior-izquierda que a pesar de que su secuencia es la cuarta sus fijaciones promedio y su tiempo de permanencia son los indicadores que le siguen. Así pues, de manera preliminar se puede decir que la zona (o área) que obtiene mayor atención visual dentro del tarjetón electoral es la posición central-izquierda, la cual consigue que no solo se observe el partido político, sino también el candidato político mientras que otras posiciones no consiguen que esto se dé de una forma balanceada dado que el participante tiende a realizar su recorrido visual o bien en el rostro del candidato, o bien en el partido político.

\section{Conclusiones preliminares}

A partir de lo obtenido, es claro que puede existir una relación entre la posición de los candidatos dentro del estímulo visual que es el tarjetón electoral y la atención visual prestada por parte de los participantes (votantes) a la hora de observar el tarjetón donde se presenta aleatorización de la posición del candidato político. No obstante, la fase preliminar tiene limitaciones en cuanto al total de resultados obtenidos, por lo cual se requiere que se obtenga una cantidad sólida de resultados (100 por tarjetón aproximadamente) y así se pueda generar de una manera más rígida y robusta un análisis directamente bajo los KPIs de las AOIs, aparte de lo visual. Este análisis terminaría por comprender una diferencia entre las medias de las fijaciones promedio de las posiciones previamente marcadas en este estudio para así determinar si efectivamente existe o no una diferencia significativa contundente entre la atención visual de una posición frente a otra dentro del estímulo. Por otra parte, a manera de futura orientación (y complemento) este estudio pretende alcanzar una comparativa directamente con la toma de decisión del votante cruzando resultados de votaciones simuladas y aleatorizadas en espacios que asemejen "urnas de votación" con los resultados de las visualizaciones aleatorizadas en el eye-tracker SMI Red $500 \mathrm{~Hz}$ del NeuroSmartLab para así tener una caracterización más completa sobre el panorama del experimento realizado.

\section{Referencias bibliográficas}

Arias-Velandia, N., Rincón-Báez, W. U., \& Cruz-Pulido, J. M. (2018). DESEMPEÑO DE MUJERES Y HOMBRES EN EDUCACIÓN SUPERIOR PRESENCIAL, VIRTUAL Y A DISTANCIA EN COLOMBIA - Women and men performance in face-to-face, virtual and distance higher education in Colombia. Panorama, 12(22), 57-69. https://doi.org/10.15765/pnrm.v12i22.1142

Alvarez, R. M., Sinclair, B., \& Hasen, R. L. (2006). How Much Is Enough? The "Ballot Order Effect" and the Use of Social Science Research in Election Law Disputes. ELECTION LAW JOURNAL, (1), 40.

Bagley, C. (1965). “Does Candidates' Position on the Ballot Paper Influence Voters' Choice? A Study of the 1959 and 1964 British General Elections". Parliamentary Affairs 19 (2): 162-174

Blom-Hansen, J., Elklit, J., Serritzlew, S., \& Villadsen, L. R. (2016). Ballot position and election results: Evidence from a natural experiment. Electoral Studies, 44, 172-183.

Brockington, D. (2003). “A Low Information Theory of Ballot Position Effect”. Political Behavior 25 (1): 1-27.

Connor, C. E., Egeth H. E., Yantis S. (2004). Visual Attention: Bottom-Up Versus Top-Down. Current Biology, Vol. 14, R850-R852. DOI 10.1016/j.cub.2004.09.041

Benítez, P., Quintero, M., Márquez, K., \& Ayala, A. O. (2015). Beneficios y garantías de los miembros de la fuerza pública en marcos de justicia transicional en perspectiva comparada: lecciones para el caso colombiano. Panorama, 9(16), $105-116$. https://doi.org/10.15765/pnrm.vgi16.630

Camejo Ruiz, A. J. (2019). La emergente ética y responsabilidad social en las relaciones laborales en el siglo XXI (1st ed.). Bogotá: Institución Universitaria Politécnico Grancolombiano.

Daza-Orozco, C. E. (2019). Iniciación científica: conceptualización, metodologías y buenas prácticas. Bogotá: Sociedad Colombiana de Investigación e Innovación Formativa - SCOIF. Retrieved from http://www.scoif.com/iniciacion-cientifica/

Dos Santos Amaro Elías; Nogales Bocio, Antonia Isabel; Pérez Calle, Begoña; Pérez Lagos, Camila; Marta-Lazo, Carmen; Barredo Ibáñez, Daniel; De La Garza Montemayor, Daniel Javier; Murcia Quiñones, Harvey; Gil Quintana, J, Jenny Juliana, A. S. L. R. P. (2019). Ciudadanías digitales: perspectivas desde los medios, el periodismo y la educomunicación (1st ed.; C.-C. M. Isabel, ed.). Bogotá: Institución Universitaria Politécnico Grancolombiano.

Faas, T., \& Schoen, H. (2006). The importance of being first: Effects of candidates' list positions in the 2003 Bavarian state election. Electoral Studies, 25(1), 91-102.

Grant, D. (2017). The ballot order effect is huge: evidence from Texas. Public Choice, 172(3-4), 421-442.

Jun, B. H., \& Min, H. (2017). What creates heterogeneity in ballot order effects? Evidence from Korea's local elections of education superintendent. Electoral Studies, 46, 1-14.Marcinkiewicz, K. (2014). Electoral contexts that assist voter 
coordination: Ballot position effects in Poland. Electoral Studies, 33, 322-334. https://doi.org/10.1016/j.electstud.2013.07.018

McMains S.A., Kastner S. (2009) Visual Attention. In: Binder M.D., Hirokawa N., Windhorst U. (eds) Encyclopedia of Neuroscience. Springer, Berlin, Heidelberg. DOI: https://doi.org/10.1007/978-3-540-29678-2_6344

Norman-Acevedo, E. (2018). Rompiendo Barreras, 10 Años de la Educación Virtual en el Politécnico Grancolombiano. (primera; Eduardo Norman-Acevedo, ed.). Retrieved from http://alejandria.poligran.edu.co/handle/10823/1146

Norman-Acevedo, E. (2019). Consumer cultural studies (1st ed.; E Norman-Acevedo, ed.). Bogotá: Institución Universitaria Politécnico Grancolombiano.

Oviedo, L. B., \& Silva, M. C. (2017). LA INVESTIGACIÓN ACCIÓN Y EL APRENDIZAJE POR PROYECTOS EN EL MARCO DEL MODELO PEDAGÓGICO ENSEÑANZA PARA LA COMPRENSIÓN. EXPERIENCIA DEL COLEGIO VISIÓN MUNDIAL EN COMUNIDADES VULNERABLES DE MONTERÍA-Action research and Project Based Learning in ... Panorama, 11(21), 38-51. https://doi.org/10.15765/pnrm.v11i21.1053

Quiroga, M. M., \& Becerra, A. (2018). El efecto de la posición del candidato en la papeleta de votación. El caso de las elecciones locales chilenas de 2008 y 2012. Colombia Internacional, (96), 29-55.

Rigo, D. Y., \& Donolo, D. (2016). Evaluación... más de los mismo desafiando formatos y modalidades sin libreto. Panorama, 10(19), 62-75. https://doi.org/10.15765/pnrm.v10i19.829

Robson, C., \& Walsh, B. (1974). The importance of positional voting bias in the Irish general election of 1973. Political Studies, 22(2), 191-203.

Serrano Marín Rodrigo; Palencia Triana, César Augusto; Merino Jara, Claudio Esteban; Silva Echeto, Víctor; Villasana López, Pedro Enrique; Jiménez Díaz, Luis, V. B. S. (2019). De los estados larvales a las mariposas: escenarios de la razón neoliberal en América Latina (1st ed.). Bogotá: Institución Universitaria Politécnico Grancolombiano.

Zabala-Leal, T. D. (2019). Mitigación del riesgo de lavado de activos y financiación del terrorismo (1st ed.). Retrieved from https://repository.poligran.edu.co/handle/10823/1909?show=full 